\title{
PD-L1 Expression in Different Segments and Histological Types of Ovarian Cancer According to Lymphocytic Infiltrate
}

\author{
Ljubiša Jovanović $^{1, *(\mathbb{D}, \text { Radmila Janković }}{ }^{2} \mathbb{D}$, Andja Ćirković ${ }^{3}$, Milena Jović ${ }^{4}$, Tijana Janjić ${ }^{5}$, Slaviša Djuričić $^{6,7}$ \\ and Svetlana Milenković ${ }^{1}$
}

check for

updates

Citation: Jovanović, L.; Janković, R.; Ćirković, A.; Jović, M.; Janjić, T.;

Djuričić, S.; Milenković, S. PD-L1

Expression in Different Segments and Histological Types of Ovarian Cancer According to Lymphocytic Infiltrate. Medicina 2021, 57, 1309. https://

doi.org/10.3390/medicina57121309

Academic Editor: Udo Jeschke

Received: 8 October 2021

Accepted: 25 November 2021

Published: 29 November 2021

Publisher's Note: MDPI stays neutral with regard to jurisdictional claims in published maps and institutional affiliations.

Copyright: (c) 2021 by the authors. Licensee MDPI, Basel, Switzerland. This article is an open access article distributed under the terms and conditions of the Creative Commons Attribution (CC BY) license (https:/ / creativecommons.org/licenses/by/ $4.0 /)$.
1 Department of Pathology and Medical Cytology, University Clinical Center of Serbia, 11000 Belgrade, Serbia; cecanaa63@gmail.com

2 Faculty of Medicine, Institute of Pathology, University of Belgrade, 11000 Belgrade, Serbia; radmila.jankovic@med.bg.ac.rs

3 Faculty of Medicine, Institute for Medical Statistics and Informatics, University of Belgrade, 11000 Belgrade, Serbia; andja.aleksic@gmail.com

4 Institute of Pathology and Forensic Medicine, Military Medical Academy, 11000 Belgrade, Serbia; alhemastro@gmail.com

5 Clinic for Gynecology and Obstetrics, University Clinical Center of Serbia, 11000 Belgrade, Serbia; tijana1002@gmail.com

6 Department of Clinical Pathology, Mother and Child Health Care Institute of Serbia "Dr. Vukan Cupic", 11070 Belgrade, Serbia; slavisa.djuricic@gmail.com

7 Faculty of Medicine, University of Banja Luka, 78000 Banja Luka, Bosnia and Herzegovina

* Correspondence: jovanovicljubisa18@gmail.com; Tel.: +381-65-8103-486

\begin{abstract}
Background and Objectives: Ovarian cancer is the leading cause of death among gynecological tumors. PD-1/PD-L1 immunoregulatory mechanism is activated in ovarian cancers. Lymphocyte infiltration is a significant factor that affects its expression. We analyzed the correlation between localization of lymphocytic infiltrate and PD-L1 expression in epithelial ovarian tumors. Materials and Methods: PD-L1 expression was analyzed in 328 subjects, 122 with epithelial ovarian carcinoma, 42 with atypical proliferative tumor, and 164 with benign epithelial ovarian tumor. Expression in central and invasive tumor parts in epithelial ovarian carcinoma was combined with the most pronounced lymphocyte reaction. Immunohistochemical analysis was performed using the tissue microarray and correlated with a set of histopathology parameters. Results: PD-L1 expression was most prominent in epithelial ovarian carcinoma with different levels of expression observed between invasive and central tumor segments. A high level of PD-L1 expression on tumor cells was more frequently present in the invasive than in the central tumor parts $(p<0.001)$ only in highgrade serous ovarian carcinoma (HGSC). There was no significant correlation between peritumoral lymphocytic infiltrate and PD-L1 expression regardless of tumor segment. In the central tumor parts of HGSC, there was a correlation of intratumoral lymphocytic infiltrate with a higher level of PD-L1 expression ( $p=0.003)$. Conclusions: The most prominent PD-L1 expression was observed in the invasive tumor parts of HGSC. Only the central parts of the HGSC exhibited significant PD-L1 expression in association with considerable intratumoral lymphocytic infiltrate.
\end{abstract}

Keywords: ovarian cancer; PD-L1; lymphocytic infiltrate

\section{Introduction}

Epithelial ovarian cancer (EOC) has the highest mortality rate among all gynecological tumors. It represents the eighth most common female cancer [1]. Due to the absence of early symptoms and screening programs, EOC is diagnosed mostly in advanced stages, with widespread metastasis and poor prognosis despite therapy. The lack of effective therapy and the frequent relapses indicates the need for new therapeutic strategies. Platinumbased chemotherapy is the main treatment for these patients. Despite new therapy, such 
as vascular endothelial growth factor (VEGF) and poly(ADP-ribose) polymerase (PARP) inhibitors, recurrences are still inevitable [2].

Suppression of the antitumor immune response is one of the crucial mechanisms by which tumor cells ensure their survival [3]. Tumors can suppress the host's immune responses by activating physiological regulatory mechanisms (checkpoints) to escape immune detection. One such significant checkpoint is programmed death protein 1 (PD-1) and its ligand, programmed death-ligand 1 (PD-L1). PD-L1 expressed on tumor cells interacts with PD-1 receptor on T lymphocytes and leads to inhibition of secretion cytokines and deactivation of effector $\mathrm{T}$ lymphocytes. Such an interaction makes tumor cells resistant to effector lymphocytes [4,5]. The PD-1/PD-L1 pathway inhibition using monoclonal antibodies has become a promising therapy in many cancers with increased survival. Ovarian cancer cells can activate the PD-1/PD-L1 immunoregulatory mechanism and make tumors more aggressive, with poor therapy response and worse prognosis [6].

Tumor-infiltrating lymphocytes (TILs) contribute to reduced tumor growth [5]. The significance of TILs and their correlation with the prognostic parameters has been the subject of many studies $[7,8]$. In advanced carcinomas with less TIL, the antitumor immune response is weak [7]. Both intratumoral and peritumoral localizations of $\mathrm{T}$ lymphocytes are associated with better outcomes, with intratumoral localization being a slightly stronger prognostic parameter [8]. Accordingly, an association between prolonged survival in ovarian carcinoma patients and prominent TIL has been shown, regardless of histological type, grade, and stage of the tumor [9]. TIL expresses an effective memory immune response that contributes to long-term antitumor immunity. Effector T lymphocytes suppress potential recurrences [10]. Therefore, antitumor immunotherapy could be more effective in EOC with prominent TIL $[7,8,10]$.

PD-L1 expression on ovarian cancer cells is induced by INF- $\gamma$ from T lymphocytes in the tumor microenvironment [11]. Prominent TIL is associated with high PD-L1 expression on ovarian cancer cells in various histology types [12,13]. Expression levels of PD-L1 marker and TIL are independent prognostic parameters for patients with ovarian carcinoma [14,15]. There is an association between prominent TIL and PD-L1 expression on tumor cells, especially in the most invasive parts of the tumor. PD-L1 expression on ovarian tumor cells of high-grade serous carcinoma (HGSC) correlates negatively with patients' survival, while prominent TIL contributes to better outcomes [16].

In this study, we analyzed the correlation between the localization of lymphocytic infiltrate and PD-L1 expression in epithelial ovarian tumors.

\section{Materials and Methods}

\subsection{Study Population}

The study included 328 subjects who underwent surgery due to epithelial ovarian tumor (EOT) in the period from 2017 to 2019 at the Clinic for Gynecology and Obstetrics, University Clinical Center of Serbia, Belgrade. The experimental group consisted of 122 subjects with epithelial ovarian carcinoma (EOC) and 42 with atypical proliferative tumor (ATP). The control group included 164 patients with benign epithelial ovarian tumor (BOT). The following parameters were recorded for each subject: patient's age, menopausal status, histological type of tumor, tumor differentiation, International Federation of Gynecology and Obstetrics (FIGO) stage, presence of lymphovascular tumor invasion, necrosis, and intratumoral and peritumoral lymphocyte infiltration. Patients with secondary and non-epithelial ovarian tumors were excluded from the study. The ethical approval was obtained from the Ethics Committee of the University Clinical Center of Serbia. Written informed consent was obtained from all patients.

\subsection{Tissue Microarray (TMA)}

The tissue microarray (TMA) method was performed using two cylinders of tissue from each paraffin block with a $3 \mathrm{~mm}$ puncture needle. The first cylinder was taken from the central part of the tumor, and the second cylinder was taken from the peripheral 
part. Both parts of the tumor were with the most pronounced lymphocyte reaction. The cylinders were moved to a recipient paraffin block, where a series of 28 tissue cylinders was formed [17]. In the first row of each block, a placental tissue was placed to serve for orientation and as a positive internal control for immunohistochemical analysis [14].

\subsection{Immunohistochemical Analysis}

Immunohistochemical staining for PD-L1 was performed on TMA sections, on the Autostainer Link 48, Agilent, Denmark. For the PD-L1 antibody, epitope unmasking was done in EnVision FLEX epitope unmasking solution pH 6.1 (K8005, Agilent, Santa Clara, CA, USA). The visualization system EnVision FLEX (Agilent) was used for immunohistochemical analysis. A monoclonal anti-human PD-L1 antibody (clone 22C3, M3653, Agilent) in a dilution of 1:30 was used as the primary antibody. The analysis was performed on EOC samples (central and peripheral tumor parts), samples from ATP tumors, and samples from the BOT. Positive tumor cells were counted on the $\times 400$ power field and their percentage from the total number of tumor cells was determined. Tumor cells usually show membrane staining for the PD-L1 antibody, with variable expression in the cytoplasm and nucleus. Any convincing partial or complete linear membrane PD-L1 staining in at least one viable tumor cell that is perceived as distinct from cytoplasmic staining we consider as a positive reaction $[18,19]$. The following score was used to describe the expression of PD-L1: negative (0) expression, without positive cells or with a single positive cell $(<1 \%)$; low $(1+)$ expression, with less than $10 \%$ positive cells; moderate (2+) expression, with $10-50 \%$ positive cells; and strong (3+) expression, with more than $50 \%$ positive cells. Tumors with moderate (2+) and strong (3+) positivity were considered as high PD-L1 expression.

\subsection{Tumor-Infiltrating Lymphocytes (TILs)}

The lymphocytic infiltrate was analyzed at whole slides before TMA constructions in the central and peripheral tumor parts and its localization was correlated with PDL1 expression on tumor cells. The presence of lymphocytic infiltrate was estimated on microscopic magnification $\times 50$ on HE tissue samples before microarray forming (Figure 1). Two categories were formed. Prominent lymphocytic infiltrate in EOCs was considered as TIL positive, while absent or rarely lymphocytes were designated as TIL negative [20].

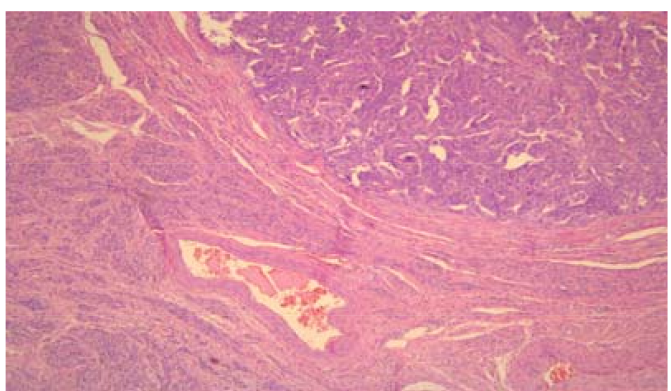

(a)

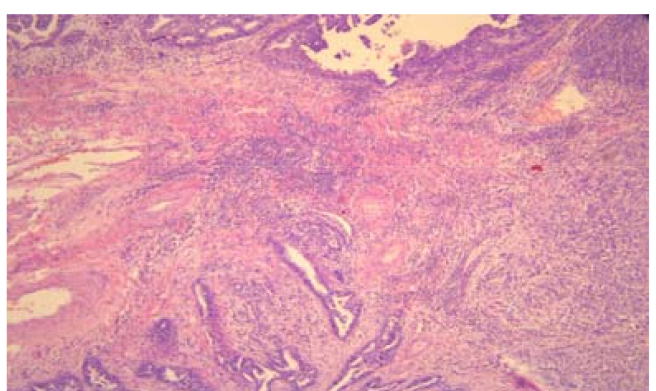

(b)

Figure 1. High-grade serous ovarian carcinoma (a) without and (b) with tumor-infiltrating lymphocytes (TILs) $(\times 50)$.

\subsection{Statistical Analysis}

Statistical analyses were performed using Statistical Package for Social Sciences 20.0 (SPSS Inc., Chicago, IL, USA). Data were expressed as means \pm standard deviation (SD) for continuous variables and percentages for categorical variables. Differences between groups for categorical data were tested by one-way ANOVA with Tuckey post hoc testing, Chi-square test or Fisher's exact test. The degree of association between categorical data was calculated using the Mann-Whitney test. The differences between central and invasive tumor parts were done by the Chi-square test. Comparison of PD-L1 expression and 
lymphocytic infiltrate type was done by the Chi-square test or Fisher's exact test. A $p$-value of less than 0.05 was considered statistically significant.

\section{Results}

\subsection{Clinical and Histopathological Characteristics}

The study included women with epithelial ovarian tumors classified into three study groups (Table 1). The mean age of all patients was $52.4 \pm 15.8$ years (age range, $15-84$ years). Women with EOC were significantly older $(p<0.001)$ than women with ATP and BOT. The vast majority of women in the EOC group were menopausal (60.7\%). The distribution of different histological types and FIGO stages is shown in Table 1.

Table 1. Demographic, clinical characteristics, and histopathological types of tumors.

\begin{tabular}{|c|c|c|c|c|c|c|c|c|}
\hline & $\begin{array}{c}\text { Total } \\
n=328\end{array}$ & $\begin{array}{c}\text { EOC } \\
n=122\end{array}$ & $\begin{array}{c}\text { EOT } \\
\text { ATP } \\
n=42\end{array}$ & $\begin{array}{c}\text { BOT } \\
n=164\end{array}$ & $p$ & $\begin{array}{c}\text { EOC vs. } \\
\text { ATP }\end{array}$ & $\begin{array}{c}\text { EOC vs. } \\
\text { BOT }\end{array}$ & $\begin{array}{c}\text { ATP vs. } \\
\text { BOT }\end{array}$ \\
\hline $\begin{array}{l}\text { Age, mean } \pm \text { sd } \\
\text { (years) }\end{array}$ & $52.4 \pm 15.8$ & $61.8 \pm 10.1$ & $45.8 \pm 12.2$ & $47.2 \pm 15.6$ & $<0.001$ & $<0.001$ & $<0.001$ & 0.834 \\
\hline \multicolumn{9}{|l|}{$\begin{array}{c}\text { Menopause, } n \\
(\%)\end{array}$} \\
\hline Yes & 199 (60.7) & $106(86.9)$ & $20(47.6)$ & $73(44.5)$ & \multirow{2}{*}{$<0.001$} & \multirow{2}{*}{$<0.001$} & \multirow{2}{*}{$<0.001$} & \multirow{2}{*}{0.718} \\
\hline No & $129(39.3)$ & $16(13.1)$ & $22(52.4)$ & $91(55.5)$ & & & & \\
\hline \multicolumn{9}{|l|}{$\begin{array}{c}\text { Histological type, } \\
n(\%)\end{array}$} \\
\hline Serous & $206(62.8)$ & $103(84.4)$ & $25(59.5)$ & $78(47.6)$ & \multirow{4}{*}{$<0.001$} & \multirow{4}{*}{$<0.001$} & \multirow{4}{*}{$<0.001$} & \multirow{4}{*}{0.044} \\
\hline Mucinous & $112(34.1)$ & $10(8.2)$ & $16(38.1)$ & $86(52.4)$ & & & & \\
\hline Endometrioid & $10(3.0)$ & $9(7.4)$ & $1(2.4)$ & $0(0.0)$ & & & & \\
\hline $\begin{array}{c}\text { FIGO stage, } n \\
(\%)\end{array}$ & & & & & & & & \\
\hline $\mathrm{I}+\mathrm{II}$ & $81(49.4)$ & $39(32.0)$ & 42 (100.0) & / & \multirow{2}{*}{$<0.001$} & \multirow{2}{*}{$<0.001$} & \multirow{2}{*}{ NA } & \multirow{2}{*}{ NA } \\
\hline III + IV & 83 (50.6) & $83(68.0)$ & $0(0.0)$ & / & & & & \\
\hline
\end{tabular}

EOT—epithelial ovarian tumor, EOC—epithelial ovarian carcinoma, ATP—atypical proliferative tumor, BOT—benign ovarian tumor, FIGO-International Federation of Gynecological and Obstetrics.

The histopathological characteristics of EOCs are presented in Table 2.

Table 2. Histopathological characteristics of EOCs.

\begin{tabular}{|c|c|c|c|c|c|c|}
\hline Characteristic & $\begin{array}{l}\text { Serous } \\
n=103\end{array}$ & $\begin{array}{c}\text { EOCs } n=122 \\
\text { Mucinous } \\
n=10\end{array}$ & $\begin{array}{c}\text { Endometrioid } \\
\qquad n=9\end{array}$ & $p^{\mathrm{a}}$ & $p^{\mathrm{b}}$ & $p^{c}$ \\
\hline \multicolumn{7}{|l|}{ Differentiation (Grade), $n(\%)$} \\
\hline $\begin{array}{l}\text { Well and moderately } \\
\text { differentiated } \\
\text { (Grade } 1+\text { Grade } 2)\end{array}$ & $12(11.7)$ & $9(90.0)$ & $6(66.7)$ & $<0.001$ & $<0.001$ & 0.213 \\
\hline $\begin{array}{c}\text { Poorly differentiated } \\
\text { (Grade 3) }\end{array}$ & 91 (88.3) & 1 (10.0) & $3(33.3)$ & & & \\
\hline Lymphovascular invasion, $n(\%)$ & $95(92.2)$ & $4(40.0)$ & $4(44.4)$ & $<0.001$ & $<0.001$ & 1.000 \\
\hline Necrosis, $n(\%)$ & $88(85.4)$ & $5(50.0)$ & $4(44.4)$ & 0.005 & 0.001 & 1.000 \\
\hline $\begin{array}{l}\text { Intratumoral lymphocyte } \\
\quad \text { infiltration, } n(\%)\end{array}$ & $89(86.4)$ & $3(30.0)$ & $7(77.8)$ & $<0.001$ & $<0.001$ & 0.070 \\
\hline $\begin{array}{l}\text { Peritumoral lymphocyte } \\
\quad \text { infiltration, } n(\%)\end{array}$ & $96(93.2)$ & $6(60.0)$ & $8(88.9)$ & 0.008 & 0.003 & 0.303 \\
\hline
\end{tabular}

${ }^{a}$ Serous vs. Mucinous, ${ }^{b}$ Serous vs. Endometrioid, ${ }^{c}$ Mucinous vs. Endometrioid. EOC-epithelial ovarian carcinoma. 


\subsection{Immunohistochemical Analysis of PD-L1 Expression}

There was a statistically significant difference in the expression level of PD-L1 between EOC, ATP tumors, and BOT. Higher expression was proven in EOC than in ATP and BOT (Table 3).

Table 3. PD-L1 expression levels in epithelial ovarian tumors.

\begin{tabular}{|c|c|c|c|c|c|c|c|}
\hline $\begin{array}{c}\text { PD-L1 Expression } \\
\text { Level }\end{array}$ & EOC & $\begin{array}{l}\text { Group } \\
\text { ATP }\end{array}$ & BOT & $p$ & EOC vs. ATP & EOC vs. BOT & ATP vs. BOT \\
\hline 0 & $0(0.0)$ & 7 (16.7) & $159(97.0)$ & \multirow{4}{*}{$<0.001$} & \multirow{4}{*}{$<0.001$} & \multirow{4}{*}{$<0.001$} & \multirow{4}{*}{$<0.001$} \\
\hline $1+$ & $34(27.9)$ & $30(71.4)$ & $5(3.0)$ & & & & \\
\hline $2+$ & $86(70.5)$ & $5(11.9)$ & $0(0.0)$ & & & & \\
\hline $3+$ & $2(1.6)$ & $0(0.0)$ & $0(0.0)$ & & & & \\
\hline
\end{tabular}

EOC—epithelial ovarian carcinoma, ATP—atypical proliferative tumor. BOT—benign ovarian tumor.

The difference in intensity of PD-L1 expression in EOCs concerning patient and tumor characteristics is presented in Table 4 . Bilateral EOCs had significantly more cases with a high intensity of PD-L1 expression $(p<0.001)$. The intensity of PD-L1 expression was higher in serous $(79.6 \%)$ than in mucinous or endometrioid EOCs $(p<0.001)$. Additionally, it was higher in HGSC than in low-grade serous carcinoma (LGSC) $(p=0.007)$. A high level of PD-L1 expression was more frequent in EOCs with stage FIGO III and IV than in those with stage FIGO I and II $(p<0.001)$. A high level of PD-L1 expression was predominant in grade 3 EOCs $(84.2 \%)(p<0.001)$. Moreover, a high intensity of PD-L1 expression level was significantly more frequent in EOCs with tumor necrosis, lymphovascular invasion, and intratumoral and peritumoral infiltration. There was no difference in the intensity of PD-L1 expression when patients were stratified according to age groups, menopausal status, and tumor size. The examples of positive membranous PD-L1 staining in the most common high-grade serous ovarian cancer cells are shown in Figure 2.

Table 4. PD-L1 expression in relation to clinical and histopathologic characteristics of EOCs.

\begin{tabular}{|c|c|c|c|c|}
\hline \multirow{2}{*}{ Characteristic } & \multirow{2}{*}{ Categories } & \multicolumn{2}{|c|}{ PD-L1 Expression } & \multirow{2}{*}{$p$} \\
\hline & & Absent/Low * & High * & \\
\hline \multirow{2}{*}{ Age } & $<65$ & $21(31.8)$ & $45(68.2)$ & \multirow[b]{2}{*}{0.291} \\
\hline & $65+$ & $13(23.2)$ & $43(76.8)$ & \\
\hline \multirow{2}{*}{ Menopausal status } & Yes & $28(26.4)$ & $78(73.6)$ & \multirow[b]{2}{*}{0.357} \\
\hline & No & $6(37.5)$ & $10(62.5)$ & \\
\hline \multirow[b]{2}{*}{ Localization } & Unilateral & $20(50.0)$ & $20(50.0)$ & \multirow[b]{2}{*}{$<0.001$} \\
\hline & Bilateral & $14(17.1)$ & $68(82.9)$ & \\
\hline \multirow{2}{*}{ EOC size (mm) } & $<80$ & $14(24.6)$ & $43(75.4)$ & \multirow[b]{2}{*}{0.445} \\
\hline & $\geq 80$ & $20(30.8)$ & $45(69.2)$ & \\
\hline \multirow{3}{*}{ Histological type } & HGSC & $15(16.5)$ & $82(79.6) \quad 76(83.5)$ & $<0.001$ \\
\hline & Mucinous & $7(70.0)$ & $3(30.0)$ & \multirow[b]{2}{*}{$<0.001$} \\
\hline & Endometrioid & $6(66.7)$ & $3(33.3)$ & \\
\hline \multirow{3}{*}{ FIGO stage } & $\mathrm{I}+\mathrm{II}$ & $30(76.9)$ & $9(23.1)$ & \multirow{3}{*}{$<0.001$} \\
\hline & III + IV & $4(4.8)$ & $79(95.2)$ & \\
\hline & 1 & $15(100.0)$ & 0 & \\
\hline \multirow[t]{2}{*}{ Differentiation (Grade) } & 2 & $4(33.3)$ & $8(66.7)$ & \multirow{2}{*}{$<0.001$} \\
\hline & 3 & $15(15.8)$ & $80(84.2)$ & \\
\hline \multirow{2}{*}{ Necrosis } & Yes & 19 (19.6) & $78(80.4)$ & \multirow[b]{2}{*}{$<0.001$} \\
\hline & No & $15(60.0)$ & $10(40.0)$ & \\
\hline \multirow{2}{*}{ Lymphovascular invasion } & Yes & $18(17.5)$ & $85(82.5)$ & \multirow[b]{2}{*}{$<0.001$} \\
\hline & No & $16(84.2)$ & $3(15.8)$ & \\
\hline \multirow{2}{*}{$\begin{array}{l}\text { Intratumoral lymphocyte } \\
\text { infiltration }\end{array}$} & Yes & $20(20.2)$ & $79(79.8)$ & \multirow{2}{*}{$<0.001$} \\
\hline & No & $14(60.9)$ & $9(39.1)$ & \\
\hline \multirow{2}{*}{$\begin{array}{l}\text { Peritumoral lymphocyte } \\
\text { infiltration }\end{array}$} & Yes & $27(24.5)$ & $83(75.5)$ & \multirow{2}{*}{0.013} \\
\hline & No & $7(58.3)$ & $5(41.7)$ & \\
\hline
\end{tabular}

EOC-epithelial ovarian carcinoma, FIGO-International Federation of Gynecological and Obstetrics. * absent/low PD-L1 expression (0 and $1+$ ), high PD-L1 expression (2+ and $3+$ ). 


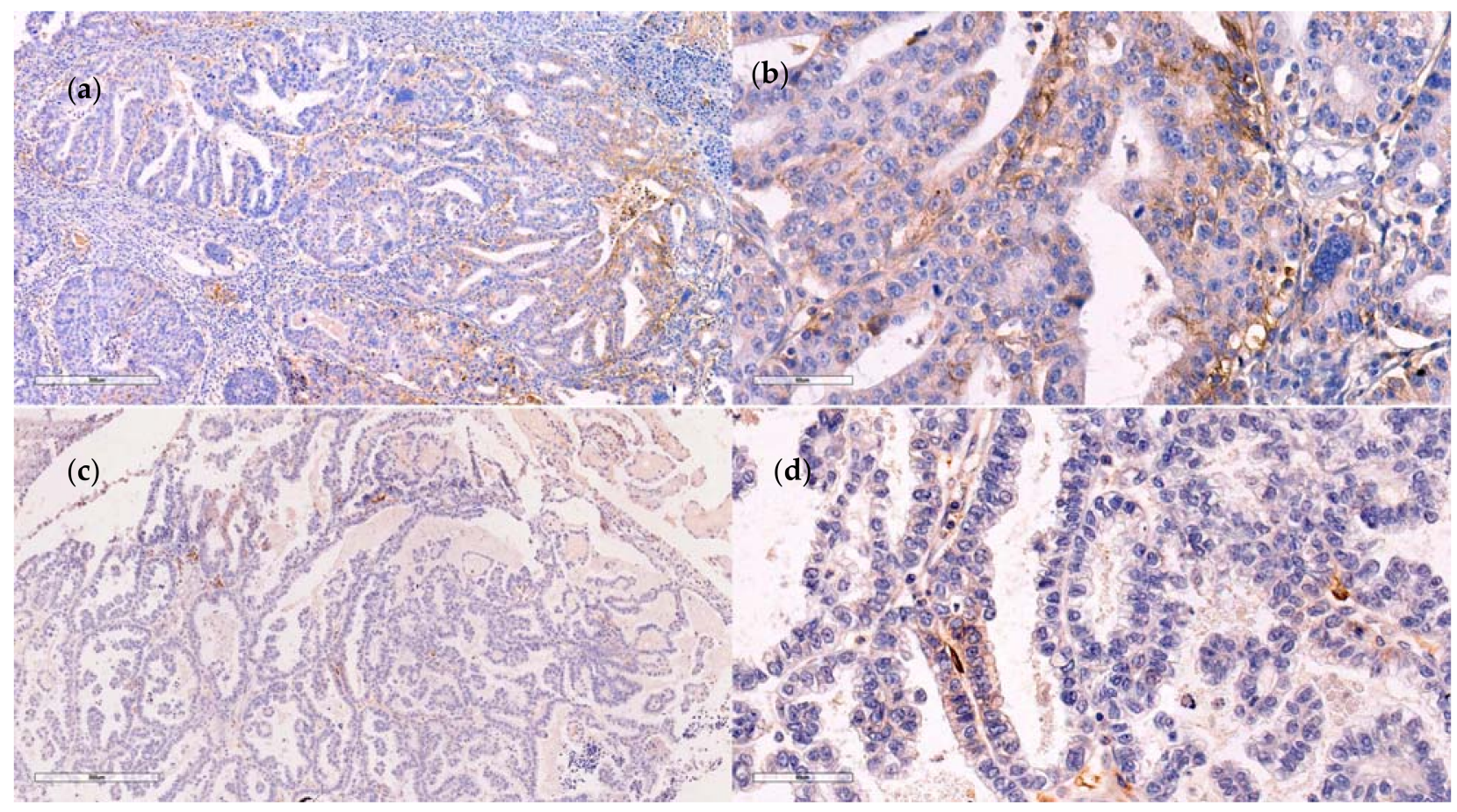

Figure 2. Different levels of PD-L1 expression in high-grade serous ovarian cancer cells. $(\mathbf{a})(\times 100)$ and $(\mathbf{b})(\times 400)$ strong PD-L1 expression, and $(\mathbf{c})(\times 100)$ and $(\mathbf{d})(\times 400)$ moderate PD-L1 expression.

3.3. PD-L1 Expression in Different Tumor Parts in Relation to the Localization of Lymphocytic Infiltrate

PD-L1 expression varied in frontal (invasive) and central tumor parts, but only in the HGSC was the statistically significant difference between these tumor parts shown $(p<0.001)$. A high level of PD-L1 expression was more frequently present in the invasive than in the central tumor parts $(p<0.001)$ (Table 5).

Table 5. PD-L1 expression in the different parts of EOC: in the center and the invasive front.

\begin{tabular}{cccc}
\hline Categories of PD-L1 Expression & \multicolumn{2}{c}{ Tumor Localization } \\
Invasive Front & $p$ \\
\hline & HGSC & \\
\hline $1+$ & $15(16.5)$ & $3(3.3)$ & \\
$2+$ & $74(81.3)$ & $60(65.9)$ & $<0.001$ \\
$3+$ & $2(2.2)$ & $28(30.8)$ & \\
LGSC & & $1(8.3)$ & 0.069 \\
$1+$ & $6(50.0)$ & $11(91.7)$ & \\
High expression (2+ and 3+) & $6(50.0)$ & $5(50.0)$ & 0.650 \\
Mucinous EOC & & $5(50.0)$ & \\
$1+$ & $7(70.0)$ & $3(33.3)$ & 0.347 \\
$2+$ & $3(30.0)$ & $6(66.7)$ & \\
Endometrioid EOC & $6(66.7)$ & & \\
$1+$ & $3(33.3)$ &
\end{tabular}

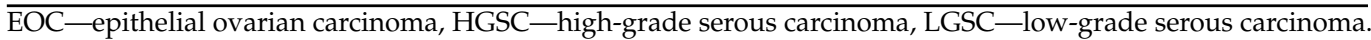

Intratumoral lymphocytic infiltrate was more frequently present $(84.3 \%)$ in the central tumor parts, with a high level of PD-L1 expression $(p=0.003)$ (Table 6). 
Table 6. PD-L1 expression categories of HGSC according to the presence of intratumoral and peritumoral lymphocytic infiltrate.

\begin{tabular}{|c|c|c|c|c|c|c|c|}
\hline \multirow{2}{*}{ Tumor Part } & \multirow{2}{*}{$\begin{array}{c}\text { Categories of PD-L1 } \\
\text { Expression }\end{array}$} & \multicolumn{2}{|c|}{$\begin{array}{l}\text { Intratumoral Lymphocytic } \\
\text { Infiltrate }\end{array}$} & \multirow[t]{2}{*}{$p$} & \multicolumn{2}{|c|}{$\begin{array}{l}\text { Peritumoral Lymphocytic } \\
\text { Infiltrate }\end{array}$} & \multirow[t]{2}{*}{$p$} \\
\hline & & with & without & & with & without & \\
\hline \multirow{2}{*}{ Center } & absent/low * & $14(15.7)$ & $7(50.0)$ & \multirow[b]{2}{*}{0.003} & $18(18.8)$ & $3(42.9)$ & \multirow[b]{2}{*}{0.126} \\
\hline & High ** & 75 (84.3) & $7(50.0)$ & & $78(81.3)$ & $4(57.1)$ & \\
\hline \multirow{2}{*}{$\begin{array}{l}\text { Invasive } \\
\text { front }\end{array}$} & absent/low * & $2(2.2)$ & $2(14.3)$ & \multirow{2}{*}{0.088} & $3(3.1)$ & 1 (14.3) & \multirow{2}{*}{0.249} \\
\hline & high * & 87 (97.8) & $12(85.7)$ & & $93(96.9)$ & $6(85.7)$ & \\
\hline
\end{tabular}

* absent/low PD-L1 expression (0 and 1+), ** high PD-L1 expression (2+ and 3+).

\section{Discussion}

PD-1/PD-L1 target therapy has recently attracted attention in EOC [2]. The efficiency of immunotherapy is based on the presence of target molecules that could be modulated by different therapeutic agents. PD-L1 receptor is one of the molecules whose functions are currently explored, especially concerning the other factors in the tumor microenvironment [14,20-22]. Immunohistochemical analysis of PD-L1 expression in tumor tissue is a prerequisite for the potential usage of PD-L1 inhibitors. Treatment outcomes should be better with this novel therapy than with standard chemotherapy agents alone $[8,14,22]$. Our study showed a significantly higher level of PD-L1 expression in EOCs than in ATP tumors or in controls, indicating that the PD-1/PD-L1 immunoregulatory mechanism is activated in EOCs, most frequently in HGSC. The distinction of PD-L1 expression between highand low-grade serous carcinomas has been described previously, as well as its association with clinical outcome [23]. These findings underline the possibility for the usage of PD-L1 inhibitors in patients with more aggressive ovarian cancers, such as HGSC.

We noted a significant difference in PD-L1 expression between central and invasive tumor parts only in the HGSC type. Other histological types of EOC did not show a significant difference. Thus, a relatively small sample of non-HGSC tumors could be related to this statistical insignificance $[18,19]$.

TILs are part of the endogenous immune reaction to the tumor cells, capable of recognition and elimination of the latter [22]. We found more pronounced TILs in HGSC with higher PD-L1 expression on tumor cells in comparison to other histological types. Higher PD-L1 expression in HGSC with more TILs can be explained as the upregulation of PD-L1 receptors on tumor cells by activated T lymphocytes [24]. Studies that analyzed the association between PD-L1 expression on tumor cells and the presence of TIL found a better prognosis in cases where carcinomas had more lymphocytic infiltrate [22,24-26].

We also demonstrated more frequent PD-L1 expression in HGSC with significant intratumoral lymphocytic infiltrate, especially in central tumor parts. Our assumption, however, that the invasive tumor parts with high PD-L1 expression correlate with the most prominent peritumoral lymphocyte infiltrate did not reach statistical significance. Some authors reported differences between peritumoral and intratumoral lymphocytes concerning PD-L1 expression in HGSC [8]. A positive correlation between intratumoral lymphocytic infiltrate and higher survival rates was found. Studies also reported a positive correlation between PD-L1 expression on tumor cells and favorable prognosis in HGSC $[8,26]$. Intraepithelial TILs were described as the most significant factor for the control of EOCs' progression [22].

On the other hand, one study reported a negative correlation between PD-L1 expression on ovarian cancer cells and the presence of intratumoral lymphocytes [27]. The negative correlation is explained as inhibition of the antitumor immune response by PD-L1 molecules on cancer cells. Additionally, the small sample size and the methodological inconsistency (clone type) could be the factors contributing to these results. The same study defined PD-L1 expression and intratumoral lymphocytes as independent prognostic factors for EOCs [27]. 
One of the limitations of our study is that we did not analyze PD-L1 expression on immune cells in the tumor microenvironment. Studies of the distribution of PD-L1 and PD-1 expression on immune cells in HGSC and their diagnostic and prognostic significance found that there was a survival benefit in cases with high macrophage PD-L1 expression [28].

One recent study investigated PD-L1, PD-1, and CD8 expressions on TILs, and PDL1 expression on ovarian cancer cells, and compared results in primary EOC with their expressions in peritoneal metastases [29]. The correlation was found only in the case of PD-L1 expression on tumor cells between primary EOC and peritoneal metastases. Marker expressions on TILs were significantly different in primary EOC compared to the peritoneal metastases. The higher PD-L1 expression on TILs was in correlation with unfavorable prognosis [29]. This observation could be explained by finding that PDL1 on TILs can suppress neighboring T lymphocytes with PD-1 receptors and indicate intratumoral immune tolerance [30]. On the contrary, some researchers stated that a high level of PD-L1 expression on TILs was a protective factor for EOC. TILs with PD-L1 molecules on the surface could increase the level of cytotoxic CD8+ T lymphocytes and therefore promote antitumor response [21]. Additional investigation of PD-L1 status on TILs, especially in association with PD-1 expression, is an important subject for further research and provision of a better understanding of the immune antitumor regulation in EOC.

Regardless of recently involved drugs in EOC treatment as Bevacizumab and Olaparib, the prognosis for these patients is still poor. Implementation of PD-L1 inhibitors should be a promising strategy in EOC treatment. However, previous studies described the weak response by PD-L1 inhibitors for these patients. Different components of the EOC microenvironment contribute to this failure [31]. Significant heterogeneity in EOC could be an additional factor for this inadequate response. In HGSC at least four genomic classes were identified differing for immunoreactivity [31,32]. Therefore, a comprehensive analysis of the genomic status of EOC and immunological characteristics in the tumor microenvironment could be predictive factors for immune therapy response [2,32]. We believe that additional analysis of interactive signaling pathways could improve strengthen of PD-L1 inhibitors. Lymphocyte activation gene (LAG-3) and a cluster of differentiation 27 (CD27) are such molecules with the immunomodulatory role that are worth further researching [32].

Recent studies have shown insufficiently reliable PD-L1 staining in EOC for the usage of PD-L1 inhibitors. Some patients without PD-L1 expression had a satisfying therapy response to PD-L1 inhibitors. On the contrary, there were patients without therapy effects despite positive PD-L1 expression. The explanation could be tumor heterogeneity, which is quite pronounced in metastatic tumor sites. It leads to different PD-L1 values suggesting immunohistochemistry analysis in both primary and metastatic tumors for the increased prognostic potential of PD-L1 marker [33].

Many trials of usage of PD-L1 inhibitors in EOC have shown limited success. Patient selection based on simultaneous PD-L1 analysis in cancer cells and evaluation of TILs could improve these results [11]. One study reported about increased efficacy of immune therapies in HGSC if they activate adequate intercellular pathways in the tumor microenvironment, including TIL [34].

Studies reported a positive correlation between TILs, PD-L1 expression on ovarian cancer cells, and the presence of breast cancer genes 1 and 2 (BRCA1 and 2) mutations. The association between these parameters could imply the potential for the usage of immune therapy in ovarian cancers with BRCA mutations [25]. The synergistic use of PARP inhibitors for these patients could be promising therapeutic strategies in the future to enhance the clinical effectiveness of immunotherapy [25,32]. PARP inhibitors induce the release of neoantigens and enhance PD-L1 expression [32]. Mismatch repair (MMR) deficiency is also represented as a mechanism of immune responsiveness [32]. Previous studies reported about the association between PD-L1 expression and microsatellite instability status [33]. 
Overall survival (OS) is worse in many cancers with high PD-L1 expression. Some studies represented the inconsistent OS outcomes of PD-L1 expression in ovarian HGSC. It could be because of various detection and scoring systems and differences in sample size. The presence of antitumor immune response as TILs predicts significantly better OS in EOC. Correlation between prolonged OS and the presence of TILs indicates that EOCs are for certain intrinsically immunogenic tumors [5].

PD-L1 expression in HGSC was approached in only a few studies with variable results [5]. There is a lot of contradiction about the PD-L1 expression on tumor cells concerning the type of lymphocytic infiltrate. In this study, we additionally analyze expression status in different tumor parts and among different histological types of EOC. Our study reports about the significance of the tumor microenvironment and antitumoral immune response, which is more expressed in advanced FIGO stages and more aggressive histological EOC types (HGSC). This study involved a large number of EOTs with different biological behaviors and histological types, which should improve the validity of the obtained results.

The analysis of PD-L1 expression with assessment TIL status could become a promising therapeutic target for patients with ovarian HGSC [5]. We need more studies to validate their therapeutic potential. The analysis of a combination of different therapy modalities could bring to better outcomes and longer OS for patients with HGSC [5].

\section{Conclusions}

We confirmed higher PD-L1 expression in an aggressive histologic type of EOC (HGSC) in advanced FIGO stages. Invasive tumor parts of HGSC showed the most frequent PD-L1 expression, but only in the central parts of HGSC there was significant PDL1 expression associated with remarkable intratumoral lymphocytic infiltrate. Our study results support the hypothesis that the PD-L1 inhibitors could be an effective therapeutic option in aggressive ovarian carcinomas as HGSC, especially with prominent intratumoral lymphocytic infiltrate.

Author Contributions: L.J.: Conceptualization, Methodology, Investigation, Resources, Data curation, Writing-Original draft preparation, and Visualization. R.J.: Methodology, Investigation, Resources, Writing-Reviewing and Editing, and Supervision. A.C..: Methodology, Investigation, Data curation, and Writing-Original draft preparation. M.J.: Methodology, Investigation, and Resources. T.J.: Writing-Original draft preparation and Visualization. S.D.: Supervision and Visualization. S.M.: Resources and Investigation. All authors have read and agreed to the published version of the manuscript.

Funding: This research received no external funding.

Institutional Review Board Statement: The study was conducted in accordance with the Declaration of Helsinki, and the protocol was approved by the Ethics Committee of University Clinical Center of Serbia and Faculty of Medicine, University of Belgrade, Serbia (747/3; 19 July 2018).

Informed Consent Statement: All subjects gave their informed consent for inclusion before they participated in the study. Written informed consent was obtained from the patients to publish this paper.

Data Availability Statement: Data available in a publicly accessible repository.

Conflicts of Interest: The authors declare no conflict of interest.

\section{References}

1. Siegel, R.L.; Miller, K.D.; Jemal, A. Cancer statistics. CA Cancer J. Clin. 2020, 70, 7-30. [CrossRef] [PubMed]

2. Battaglia, A.; Piermattei, A.; Buzzonetti, A.; Pasciuto, T.; Zampetti, N.; Fossati, M.; Angelico, G.; Iacobelli, V.; Nero, C.; Iannucci, V.; et al. PD-L1 Expression on Circulating Tumour-Derived Microvesicles as a Complementary Tool for Stratification of High-Grade Serous Ovarian Cancer Patients. Cancers 2021, 13, 5200. [CrossRef] [PubMed]

3. Motalleb Nezhad, M.; Jadidi-Niaragh, F.; Ghamsari, E.S.; Bagheri, S.; Gharibi, T.; Yousefi, M. The immunobiology of myeloidderived suppressor cells in cancer. Tumor Biol. 2016, 37, 1387-1406. [CrossRef] [PubMed] 
4. Chen, D.S.; Mellman, I. Elements of cancer immunity and the cancer-immune set point. Nature 2017, 541, 321-330. [CrossRef] [PubMed]

5. Farrag, M.S.; Abdelwahab, K.; Farrag, N.S.; Elrefaie, W.E.; Emarah, Z. Programmed death ligand-1 and CD8 tumor-infiltrating lymphocytes (TILs) as prognostic predictors in ovarian high-grade serous carcinoma (HGSC). J. Egypt. Natl. Cancer Inst. 2021, 33, 16. [CrossRef]

6. Pietzner, K.; Nasser, S.; Alavi, S.; Darb-Esfahani, S.; Passler, M.; Muallem, M.Z.; Sehouli, J. Checkpoint-inhibition in ovarian cancer: Rising star or just a dream? J. Gynecol. Oncol. 2018, 29, e93. [CrossRef]

7. Zhu, X.; Lang, J. Programmed death-1 pathway blockade produces a synergistic antitumor effect: Combined application in ovarian cancer. J. Gynecol. Oncol. 2017, 28, e64. [CrossRef]

8. Darb-Esfahani, S.; Kunze, C.A.; Kulbe, H.; Sehouli, J.; Wienert, S.; Lindner, J.; Budczies, J.; Bockmayr, M.; Dietel, M.; Denkert, C.; et al. Prognostic impact of programmed cell death-1 (PD-1) and PD-ligand 1 (PD-L1) expression in cancer cells and tumorinfiltrating lymphocytes in ovarian high grade serous carcinoma. Oncotarget 2016, 7, 1486-1499. [CrossRef]

9. Hwang, W.T.; Adams, S.F.; Tahirovic, E.; Hagemann, I.S.; Coukos, G. Prognostic significance of tumor-infiltrating T cells in ovarian cancer: A meta-analysis. Gynecol. Oncol. 2012, 124, 192-198. [CrossRef]

10. Landskron, J.; Helland, O.; Torgersen, K.M.; Aandahl, E.M.; Gjertsen, B.T.; Bjorge, L.; Taskén, K. Activated regulatory and memory T-cells accumulate in malignant ascites from ovarian carcinoma patients. Cancer Immunol. Immunother. 2015, 64, 337-347. [CrossRef]

11. Bansal, A.; Srinivasan, R.; Rohilla, M.; Rai, B.; Rajwanshi, A.; Suri, V.; Saha, S.C. Immunotyping in tubo-ovarian high-grade serous carcinoma by PD-L1 and CD8+ T-lymphocytes predicts disease-free survival. APMIS 2021, 129, 254-264. [CrossRef]

12. D’Incecco, A.; Andreozzi, M.; Ludovini, V.; Rossi, E.; Capodanno, A.; Landi, L.; Tibaldi, C.; Minuti, G.; Salvini, J.; Coppi, E.; et al. PD-1 and PD-L1 expression in molecularly selected non-small-cell lung cancer patients. Br. J. Cancer 2015, 112, 95-102. [CrossRef]

13. Heymann, J.J.; Bulman, W.A.; Swinarski, D.; Pagan, C.A.; Crapanzano, J.P.; Haghighi, M.; Fazlollahi, L.; Stoopler, M.B.; Sonett, J.R.; Sacher, A.G.; et al. PD-L1 expression in non-small cell lung carcinoma: Comparison among cytology, small biopsy, and surgical resection specimens. Cancer Cytopathol. 2017, 125, 896-907. [CrossRef]

14. Scognamiglio, G.; De Chiara, A.; Di Bonito, M.; Tatangelo, F.; Losito, N.S.; Anniciello, A.; De Cecio, R.; D’Alterio, C.; Scala, S.; Cantile, M.; et al. Variability in Immunohistochemical Detection of Programmed Death Ligand 1 (PD-L1) in Cancer Tissue Types. Int. J. Mol. Sci. 2016, 17, 790. [CrossRef]

15. Gaillard, S.L.; Secord, A.A.; Monk, B. The role of immune checkpoint inhibition in the treatment of ovarian cancer. Gynaecol. Oncol. Res. Pract. 2016, 3, 11. [CrossRef]

16. Wang, Q.; Lou, W.; Di, W.; Wu, X. Prognostic value of tumor PD-L1 expression combined with CD8 ${ }^{+}$tumor infiltrating lymphocytes in high grade serous ovarian cancer. Int. Immunopharmacol. 2017, 52, 7-14. [CrossRef]

17. Choi, C.H.; Kim, K.H.; Song, J.Y.; Choi, S.J.; Kim, L.; Park, I.S.; Han, J.Y.; Kim, J.M.; Chu, Y.C. Construction of high-density tissue microarrays at low cost by using self-made manual microarray kits and recipient paraffin blocks. Korean J. Pathol. 2012, 46, 562-568. [CrossRef]

18. Dako. PD-L1 IHC 22C3 PharmDx Interpretation Manual-Gastric or Gastroesophageal Junction Adenocarcinoma. PD-L1 IHC 22C3 pharmDx Is FDA-Approved for In Vitro Diagnostic Use. 2019. Available online: https://www.agilent.com/cs/library/ usermanuals / public/29219_pd-11-ihc-22C3-pharmdx-gastric-interpretation-manual_us.pdf (accessed on 28 November 2021).

19. Dako. PD-L1 IHC 22C3 PharmDX Interpretation Manual-NSCLC—For In Vitro Diagnostic Use. 2018. Available online: https: / / usermanual.wiki/m/36cf5deff6015d44ebe696b439deba5ec850657a9b00689e6c52d5bc11706076.pdf (accessed on 28 November 2021).

20. Teng, M.W.; Ngiow, S.F.; Ribas, A.; Smyth, M.J. Classifying cancers based on T-cell infiltration and PD-L1. Cancer Res. 2015, 75, 2139-2145. [CrossRef]

21. Zhang, C.; Yang, Q. Predictive Values of Programmed Cell Death-Ligand 1 Expression for Prognosis, Clinicopathological Factors, and Response to Programmed Cell Death-1/Programmed Cell Death-Ligand 1 Inhibitors in Patients with Gynecological Cancers: A Meta-Analysis. Front. Oncol. 2021, 10, 572203. [CrossRef]

22. Santoiemma, P.P.; Powell, D.J., Jr. Tumor infiltrating lymphocytes in ovarian cancer. Cancer Biol. Ther. 2015, 16, 807-820. [CrossRef]

23. Nhokaew, W.; Kleebkaow, P.; Chaisuriya, N.; Kietpeerakool, C. Programmed Death Ligand 1 (PD-L1) Expression in Epithelial Ovarian Cancer: A Comparison of Type I and Type II Tumors. Asian Pac. J. Cancer Prev. 2019, 20, 1161-1169. [CrossRef]

24. Webb, J.R.; Milne, K.; Kroeger, D.R.; Nelson, B.H. PD-L1 expression is associated with tumor-infiltrating T cells and favorable prognosis in high-grade serous ovarian cancer. Gynecol. Oncol. 2016, 141, 293-302. [CrossRef]

25. Strickland, K.; Howitt, B.E.; Rodig, S.J.; Ritterhouse, L.; D'Andrea, D.A.; Matulonis, U.; Konstantinopoulos, P. Tumor infiltrating and peritumoral T cells and expression of PD-L1 in BRCA1/2-mutated high grade serous ovarian cancers. J. Clin. Oncol. 2015, 33, 5512. [CrossRef]

26. Chen, H.; Molberg, K.; Strickland, A.L.; Castrillon, D.H.; Carrick, K.; Jiang, Q.; Niu, S.; Rivera-Colon, G.; Gwin, K.; Hinson, S.; et al. PD-L1 Expression and CD8+ Tumor-infiltrating Lymphocytes in Different Types of Tubo-ovarian Carcinoma and Their Prognostic Value in High-grade Serous Carcinoma. Am. J. Surg. Pathol. 2020, 44, 1050-1060. [CrossRef]

27. Hamanishi, J.; Mandai, M.; Iwasaki, M.; Okazaki, T.; Tanaka, Y.; Yamaguchi, K.; Higuchi, T.; Yagi, H.; Takakura, K.; Minato, N.; et al. Programmed cell death 1 ligand 1 and tumor-infiltrating CD8+ T lymphocytes are prognostic factors of human ovarian cancer. Proc. Natl. Acad. Sci. USA 2007, 104, 3360-3365. [CrossRef] 
28. Martin de la Fuente, L.; Westbom-Fremer, S.; Arildsen, N.S.; Hartman, L.; Malander, S.; Kannisto, P.; Måsbäck, A.; Hedenfalk, I. PD-1/PD-L1 expression and tumor-infiltrating lymphocytes are prognostically favorable in advanced high-grade serous ovarian carcinoma. Virchows Arch. 2020, 477, 83-91. [CrossRef]

29. Bekos, C.; Pils, D.; Dekan, S.; Hofstetter, G.; Horak, P.; Reinthaller, A.; Polterauer, S.; Schwameis, R.; Aust, S. PD-1 and PD-L1 expression on TILs in peritoneal metastases compared to ovarian tumor tissues and its associations with clinical outcome. Sci. Rep. 2021, 11, 6400. [CrossRef]

30. Diskin, B.; Adam, S.; Cassini, M.F.; Sanchez, G.; Liria, M.; Aykut, B.; Buttar, C.; Li, E.; Sundberg, B.; Salas, R.D.; et al. PD-L1 engagement on $\mathrm{T}$ cells promotes self-tolerance and suppression of neighboring macrophages and effector T cells in cancer. Nat. Immunol. 2020, 21, 442-454. [CrossRef]

31. Pawłowska, A.; Kwiatkowska, A.; Suszczyk, D.; Chudzik, A.; Tarkowski, R.; Barczyński, B.; Kotarski, J.; Wertel, I. Clinical and Prognostic Value of Antigen-Presenting Cells with PD-L1/PD-L2 Expression in Ovarian Cancer Patients. Int. J. Mol. Sci. 2021, 22, 11563. [CrossRef]

32. Maiorano, B.A.; Maiorano, M.F.P.; Lorusso, D.; Maiello, E. Ovarian Cancer in the Era of Immune Checkpoint Inhibitors: State of the Art and Future Perspectives. Cancers 2021, 13, 4438. [CrossRef]

33. Parvathareddy, S.K.; Siraj, A.K.; Al-Badawi, I.A.; Tulbah, A.; Al-Dayel, F.; Al-Kuraya, K.S. Differential expression of PD-L1 between primary and metastatic epithelial ovarian cancer and its clinico-pathological correlation. Sci. Rep. 2021, 11, 3750. [CrossRef] [PubMed]

34. Wan, C.; Keany, M.P.; Dong, H.; Al-Alem, L.F.; Pandya, U.M.; Lazo, S.; Boehnke, K.; Lynch, K.N.; Xu, R.; Zarrella, D.T.; et al. Enhanced Efficacy of Simultaneous PD-1 and PD-L1 Immune Checkpoint Blockade in High-Grade Serous Ovarian Cancer. Cancer Res. 2021, 81, 158-173. [CrossRef] [PubMed] 